\title{
Sortilin and Homocysteine as Potential Biomarkers for Coronary Artery Diseases
}

\author{
Rehab H Werida ${ }^{\prime}$ \\ Ayman Omran ${ }^{2}$ \\ Noha M El-Khodary (D) \\ 'Department of Clinical Pharmacy and \\ Pharmacy Practice, Faculty of Pharmacy, \\ Damanhour University, Damanhour, \\ Egypt; ${ }^{2}$ Department of Cardiology, \\ Damanhour National Medical Institute, \\ Damanhour, Egypt
}

Purpose: The aim of this study was to assess the relationship of coronary artery disease (CAD) with levels of homocysteine and sortilin in Egyptian patients.

Background: CAD is a primary contributor to cardiac disease and a prominent cause of death globally.

Patients and Methods: We enrolled 45 patients with CAD evaluated by coronary CT angiography and 42 control subjects without CAD. Plasma-homocysteine and -sortilin levels were measured with a commercial ELISA kit.

Results: Elevated levels of homocysteine and sortilin were observed in the CAD patients compared to controls $(13.75 \pm 1.40$ vs $7.73 \pm 2.06 \mu \mathrm{mol} / \mathrm{L}, P=0$ and $160.91 \pm 32.17$ vs 143.02 $\pm 32.30 \mathrm{ng} / \mathrm{dL}, P=0.02$, respectively). Significantly higher total cholesterol, low densitylipoprotein cholesterol and triglycerides $(P<0.05)$ and lower high density-lipoprotein cholesterol $(P<0.05)$ were seen among patients with CAD than the control group. Sortilin levels were positively associated with homocysteine levels $(r=0.32, P=0.006)$, total cholesterol $(r=0.61, P=0)$, low density-lipoprotein cholesterol $(r=0.37, P=0.001)$, triglycerides $(r=0.91, P=0)$, troponin I $(r=0.82, P=0)$, Gensini score $(r=0.93, P=0)$ and high-sensitivity CRP $(r=0.87, P=0)$ in all subjects. Homocysteine has a significantly negative association with high density-lipoprotein cholesterol $(r=-0.42, P=0)$.

Conclusion: Elevated homocysteine and sortilin levels are crucial risk factors of CAD in Egyptian patients.

Keywords: sortilin, homocysteine, coronary artery disease

\section{Introduction}

Coronary heart diseases (CHDs), such as angina, stroke, and myocardial infarction, are serious atherosclerotic conditions leading to death. Atherosclerosis is an inflammatory condition of vessels characterized by atheroma (or plaque) buildup through thickening of intimae with accumulation of cholesterol and macrophage foam-cell induction on injured blood-vessel walls. ${ }^{1}$

Dyslipidemia, hypertension, diabetes mellitus, age, sex, smoking, and obesity are traditional risk factors of developing coronary artery disease (CAD). ${ }^{2}$ Identification of new biomarkers for the prediction of CAD is helpful in identifying high-risk CAD patients. ${ }^{3}$ Homocysteine (Hcy) is a sulfur amino acid whose metabolism stands at the intersection of two pathways: remethylation to methionine, which requires folate and vitamin $\mathrm{B}_{12}$, and transsulfuration to cystathionine, which requires pyridoxal 5'-phosphate. ${ }^{4}$

Metabolic pathway disturbance results in accumulation of intracellular Hcy, which is transferred into the plasma before reaching cytotoxic concentrations.
Correspondence: Noha M El-Khodary Department of Clinical Pharmacy and Pharmacy Practice, Faculty of Pharmacy, Damanhour University, Damanhour, Egypt Tel +201273740276

Fax +20 45 3334595

Email noha.mahmoud@pharm.dmu.edu. eg; nohamahmoud_55@hotmail.com 
Hyperhomocysteinemia is caused by disease states, nutritional deficiencies, genetic mutations, and medications, due to alteration of the pathway of its metabolism. ${ }^{5}$ Increased rates of cardiovascular events are correlated with high levels of Hcy, which has been suggested to be causatively linked to atherosclerosis. ${ }^{6}$

Large research programs have focused on the identification of new risk factors to prevent CAD, with special attention paid to Hcy, as hyperhomocysteinemia contributes to atherosclerosis through several mechanisms, such as endothelial dysfunction, increased permeability of lipid and inflammatory cells, lipoprotein oxidation, vascular inflammation, and smooth-muscle proliferation. ${ }^{7}$

Many inflammatory markers, eg, CRP, are well known to play a role in the pathogenesis of atherosclerosis, and have been associated with the risk of cardiovascular disease (CVD) ${ }^{8}$ Evidence suggests that $\mathrm{CRP}$ might have biological impacts on endothelial function, coagulation, fibrinolysis, oxidation of LDL, and plaque stability. ${ }^{9}$ Increased risk of additional coronary events in patients with CAD has been reported to be associated with highsensitivity CRP (hs-CRP) levels, ${ }^{10}$ and hs-CRP is a strong predictor of cardiovascular events. ${ }^{11}$

Detection of cardiac troponins has grown from being just a diagnostic tool for acute coronary syndrome to a biomarker for risk stratification in individuals without known CVD. ${ }^{12,13}$

Elevated high-sensitivity troponin I is substantially linked to an increase in worldwide CVD incidence in the general population independently of traditional risk factors. ${ }^{14}$

High-sensitivity troponin testing may be useful in detecting CHD and triggering more aggressive primary prevention. This indicates a paradigm change from troponin's use as a diagnostic tool in the acute setting to its use as a main preventive strategy. ${ }^{12}$

Sortilin has recently received much interest, owing to its suspected roles in lipid-disorder diseases. ${ }^{15}$ Sotilin, a novel regulator of lipid metabolism, is coded by the cardiovascular-risk gene SORT1 and is likely to facilitate the development of atherosclerosis by affecting lipid metabolism in the liver and macrophages. In atherosclerotic lesions, sortilin has been reported to be overexpressed, but is rarely detectable in normal young vessels, which further indicates that it contributes to and supports the progression of atherosclerosis. ${ }^{15-18}$

The proatherosclerotic mechanism of sortilin is due to its ability to affect liver- and macrophage-lipid metabolism, leading to increased plasma levels of lowdensity lipoprotein cholesterol (LDL-C) and macrophagelipid accumulation. On the other hand, sortilin accelerates the biosynthesis and release of very low-density lipoprotein (VLDL) from the liver, leading to elevated plasma LDL-C levels and atherosclerosis development. ${ }^{16,19}$

Sortilin inhibits hepatic uptake of plasma LDL-C, resulting in elevated plasma LDL-C levels and atherosclerosis development. ${ }^{20}$ Sortilin overexpression in macrophages can promote the uptake of LDL-C and intracellular lipid accumulation, which results in the formation of foam cells and atherosclerotic lesion development. ${ }^{21}$ In this study, we studied whether circulating Hcy and sortilin can be used as biomarkers for screening of CAD in Egyptian population. These biomarkers may be good candidates as therapeutic targets, leading to obviation of complications and decreasedmortality rates, and could help in developing novel therapeutic strategies to stop atherosclerosis progression caused by hyperlipidemia.

\section{Patients and Methods Study Population}

A total of 45 CAD patients (men and women aged 35-65 years) were selected from the Cardiology Department outpatient clinics at Damanhour National Medical Institute, Egypt between June 2020 to September 2020. CAD was diagnosed by coronary angiography revealing $>70 \%$ stenosis in at least one vessel, and all CAD patients were managed with low-dose aspirin ( $\leq 100 \mathrm{mg}$ /day). A total of 42 non-CAD subjects (men and women aged 35-65 years) were also recruited as controls from outpatient clinics of the same institute during routine checkups or scheduled pharmacy refill appointments. The study was approved by the institutional ethics committee in accordance with the Declaration of Helsinki and its amendments, and all participants gave informed consent before initiation of the study. Patients with fever, bacterial/viral infection, history of renal failure, chronic hepatic diseases, arthritis, malignancies, autoimmune disease, or other severe medical illnesses were excluded. Patients who had taken any medication containing vitamins or folic acid in the 3 months before enrollment were also excluded.

\section{Blood Sampling}

Blood samples were obtained from each participant after fasting not less than 8 hours. For CAD patients, biochemical analysis was performed within 3 months of coronary 
CT angiography. Venous blood $(10 \mathrm{~mL})$ was withdrawn in an EDTA tube, then centrifuged at 3,000 rpm for $15 \mathrm{~min}$ at $4^{\circ} \mathrm{C}$. The plasma was immediately frozen at $-20^{\circ} \mathrm{C}$ until analysis. The samples were evaluated in blocks to decrease interassay variability.

\section{Anthropometric Assessment}

Weight and height of the enrolled patients were assessed while patients were wearing casual clothes and barefoot. BMI was also calculated.

\section{Coronary Angiography and Blood-Pressure Measurement}

The blood pressure of the patients was measured following a 15-minute resting period in the sitting position. All patients underwent diagnostic coronary angiography through radial or femoral access. The Gensini score was utilized to determine CAD severity by an expert cardiologist who was blind to the biochemical results, ${ }^{22}$ and was calculated by multiplying the luminal narrowing of main coronary artery grading by the site and importance of the lesion factor. Luminal narrowing scores were 32 for total occlusion, 16 for $91 \%-99 \%$ stenosis, 8 for $76 \%-90 \%$ stenosis, 4 for $51 \%-75 \%$ stenosis, 2 for $26 \%-50 \%$ stenosis, and 1 for $\leq 25 \%$ stenosis. The location factor was 1.5 for a mid lesion, 1 for distal LAD (left anterior descending), mid-distal LCX (left circus flex), or RCA (right coronary artery), 5 for left main, and 2.5 for proximal lesions of LAD or LCX. Then, the sum of coronary artery scores was used to calculate the total Gensini score. $^{23}$

\section{Biochemical Analyses}

Plasma glucose was determined using the hexokinase method. Fasting lipid profile was determined using commercial kits. Total cholesterol (TC) and triglyceride (TG) levels were estimated using enzymatic colorimetry. High-density lipoprotein cholesterol (HDL-C) was determined using the precipitation method. LDL-C was calculated by the Friedewald formula: LDL-C $=[\mathrm{TC}-$ HDL-C - (TGs/5)]. hs-CRP was assessed using latexenhanced turbidometric immunoassays (Denka Seiken, Tokyo, Japan). Circulating plasma sortilin and Hcy were measured with an ELISA kit (Cosmo Bio, Carlsbad, CA, USA). For Hcy and sortilin, intra- and interassay coefficients of variation were $<10 \%$ and $<12 \%$, respectively. Sensitivity was calculated to be
$0.2-15 \mu \mathrm{mol} / \mathrm{L}$ for Hcy and $30-2,000 \mathrm{pg} / \mathrm{mL}$ for sortilin. For each patient, serum levels were measured twice and the results were averaged.

\section{Statistical Analysis}

The required sample size was calculated using $\mathrm{G}^{*}$ Power 3.1.9.7 (Institut für Experimentelle Psychologie, Heinrich Heine Universität, Dfüsseldorf, Germany). It was estimated that a sample of 74 patients would have a power of $95 \%$ to detect a medium-large effect size of 0.85 in outcome measures.

Statistical analyses were done using SPSS 25.0. Data were tested for normality using the Kolmogorov-Smirnov test. ${ }^{24}$ Fisher's exact or $\# \chi^{2}$ tests (two-sided) were used to compare qualitative data, which are described as numbers and percentages.

Continuous variables are expressed as means \pm SD and categorical variables as numbers and percentages. Comparisons between the groups were made with $t$-tests for parametric statistics. Correlations between variables were calculated using Pearson's correlations. Area under the receiver-operating characteristic (ROC) curve (AUC) was used to evaluate the measured variables predictive power for CAD. $P<0.05$ was considered statistically significant.

\section{Results}

\section{Participant Characteristics}

Figure 1 shows that 74 participants, 39 CAD patients, and 35 controls completed the study and were included in the final assessment. Participants were aged 35-65 years (45.28 \pm 6.13 and $43.94 \pm 4.61$ years for the $\mathrm{CAD}$ and control groups, respectively), and $66.22 \%$ of the study population were men. Demographic and biological characteristics of the study participants are summarized in Table 1.

Both groups were comparable in terms of age, sex, BMI, systolic, and diastolic blood pressure. The prevalence of traditional risk factors was similar between the groups, ie, the CAD group had high percentages of hypertension, diabetes, and frequency of smoking, with no significant differences compared to control group $(P>0.05)$.

\section{Circulating Homocysteine and Sortilin Concentrations}

The present study demonstrated that the plasma concentrations of Hcy were significantly higher in the CAD patients than controls $(13.75 \pm 1.40$ vs $7.73 \pm 2.06 \mu \mathrm{mol} / \mathrm{L}$, $P=0)$. Significantly greater sortilin concentrations were 


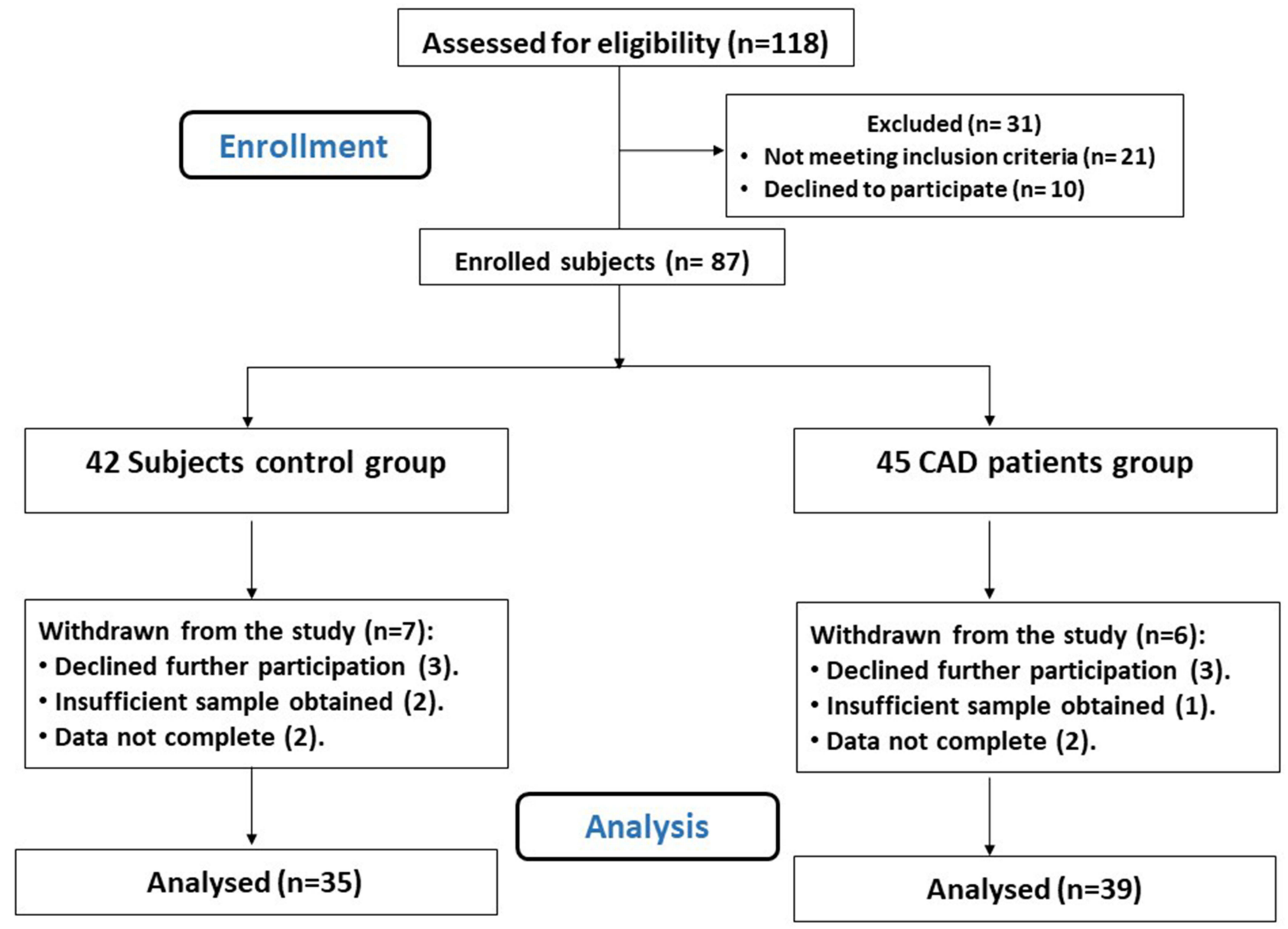

Figure I Flow diagram of the study.

observed in CAD patients than controls $(160.91 \pm 32.17$ vs $143.02 \pm 32.30 \mathrm{ng} / \mathrm{dL}, P=0.02$ ), as shown in Table 1 and Figure 2.

\section{Troponin I, hs-CRP, and Mean Angiographic Gensini Scores}

Patients with CAD had significantly higher levels of serum troponin I $(P=0.005)$, hs-CRP $(P=0)$, and mean angiographic Gensini scores $(P=0)$ than controls, as shown in Table 1 and Figure 2.

Figure 3 shows the capability of biomarkers in predicting the presence of CAD. Cutoff values of ROC curves were used to calculate diagnostic sensitivity, diagnostic specificity, and AUC of the measured biomarkers. We observed that Hcy was the most sensitive predictor (AUC 0.995, $P=0$ ), followed by LDL-C (AUC 0.803, $P=0$ ), hs-CRP (AUC 0.750, $P=0$ ), Gensini score (AUC 0.719, $P=0.001$ ), and troponin I (AUC 0.686,
$P=0.006$ ), whereas sortilin was the least sensitive (AUC 0.650, $P=0.027$ ).

\section{Associations Between Circulating Sortilin and Homocysteine}

We observed positive correlations between plasma-sortilin levels and plasma TC $(r=0.61, P=0)$, LDL-C $(r=0.37$, $P=0.001)$, TGs $(r=0.91, P=0)$, troponin I $(r=0.82, P=0)$, hs-CRP ( $r=0.87, P=0)$, Gensini score $(r=0.93, P=0)$, and plasma Hcy levels $(r=0.32, P=0.006)$ in all participants. In contrast, a significant negative association was observed between Hcy and HDL-C $(r=-0.42, P=0)$ and an insignificant negative association between sortilin and HDL-C ( $r=$ $-0.21, P=0.08$ ), as demonstrated in Table 2 .

Table 3 shows the logistic regression model used to determine whether Hcy and sortilin levels were associated with CAD. Our findings showed that Hcy $(P=0.025)$ and sortilin $(P=0.025)$ are independent risk factors of CAD. 
Table I Demographic and Biochemical Characteristics of Subjects

\begin{tabular}{|c|c|c|c|}
\hline & $\begin{array}{l}\text { Control Group } \\
\qquad(n=35)\end{array}$ & $\begin{array}{c}\text { CAD Group } \\
(n=39)\end{array}$ & $P$ \\
\hline Age (years) & $43.94 \pm 4.6 I$ & $45.28 \pm 6.13$ & 0.30 \\
\hline Sex, male/female & $23 / 12$ & $26 / 13$ & $1.00^{\mathrm{a}}$ \\
\hline BMI $\left(\mathrm{kg} / \mathrm{m}^{2}\right)$ & $32.39 \pm 1.52$ & $32.86 \pm 1.79$ & 0.22 \\
\hline $\mathrm{SBP}(\mathrm{mmHg})$ & $127.66 \pm 10.44$ & $130.23 \pm 7.44$ & 0.22 \\
\hline $\mathrm{DBP}(\mathrm{mmHg})$ & $82.11 \pm 4.27$ & $81.74 \pm 3.66$ & 0.70 \\
\hline Smokers, n (\%) & $12(34.3)$ & $18(46.2)$ & $0.35^{\mathrm{a}}$ \\
\hline Statin use, n (\%) & $17(48.6)$ & $23(59.0)$ & $0.48^{\mathrm{a}}$ \\
\hline Aspirin use, $n(\%)$ & $10(28.6)$ & $18(46.2)$ & $0.15^{\mathrm{a}}$ \\
\hline Hypertension, n (\%) & $10(28.6)$ & $12(30.8)$ & $1.00^{\mathrm{a}}$ \\
\hline Diabetic, n (\%) & $10(28.6)$ & $14(30.8)$ & $0.62^{\mathrm{a}}$ \\
\hline $\mathrm{TC}(\mathrm{mg} / \mathrm{dL})$ & $164.06 \pm 22.65$ & $|90.32 \pm| 8.5 \mid$ & $0^{*}$ \\
\hline LDL-C (mg/dL) & $102.52 \pm 21.35$ & $123.28 \pm 15.43$ & $0 *$ \\
\hline HDL-C (mg/dL) & $34.11 \pm 1.79$ & $32.57 \pm 1.61$ & $0^{*}$ \\
\hline TGs (mg/dL) & $135.74 \pm 21.00$ & $156.77 \pm 24.85$ & $0 *$ \\
\hline $\mathrm{Hs}-\mathrm{CRP}(\mathrm{mg} / \mathrm{L})$ & $6.07 \pm 0.89$ & $7.12 \pm 1.20$ & $0 *$ \\
\hline Troponin I (ng/mL) & $1.34 \pm 0.22$ & $1.49 \pm 0.22$ & $0.005^{*}$ \\
\hline Gensini score & $1.24 \pm 0.26$ & $1.50 \pm 0.25$ & $0 *$ \\
\hline $\begin{array}{l}\text { Homocysteine } \\
(\mu \mathrm{mol} / \mathrm{L})\end{array}$ & $7.73 \pm 2.06$ & $13.75 \pm 1.40$ & $0^{*}$ \\
\hline Sortilin (ng/dL) & $143.02 \pm 32.30$ & $160.91 \pm 32.17$ & $0.02 *$ \\
\hline
\end{tabular}

Notes: Data expressed as means \pm SD or $\mathrm{n}(\%)$. ${ }^{\mathrm{a}} \chi^{2}$ or Fisher's exact test (twosided); $* p<0.05$ (independent $t$-test).

Abbreviations: BMI, body-mass index; SBP, systolic blood pressure; DBP, diastolic blood pressure; TC, total cholesterol; LDL-C, low density-lipoprotein cholesterol; HDL-C, high density-lipoprotein cholesterol; TGs, triglycerides; Hs-CRP, highsensitivity CRP.

According to the logistic regression analysis in Table 4, smoking, statin use, aspirin use, diabetes, and hypertension had no significant effect in either group.

\section{Discussion}

In many countries, $\mathrm{CAD}$ is a widespread cause of death, and the detection of associated risk factors could assist in its prevention. ${ }^{25}$ Major attempts have been made in the last few years to improve antithrombotic therapy and revascularization strategies and decrease cardiac mortality, particularly in patients with acute coronary syndrome. ${ }^{26}$ However, in some high-risk patients, suboptimal results are still found. ${ }^{27-29}$ As such, the discovery of new biomarkers aimed at preventing CAD has been given considerable attention. Our study showed that plasma-Hcy concentration was significantly elevated in CAD patients compared to controls.

Since 1969, when McCully demonstrated that there was a link between high plasma concentration of Hcy and arteriosclerosis, great focus have been centered on Hcy. ${ }^{30}$ Consistently, Clarke et al showed in his research that hyperhomocysteinemia was an independent vascular and CHD risk factor. ${ }^{31}$ Similar results were found by Klerk et al in a large meta-analysis supporting the hyperhomocysteinemia and CVD association. ${ }^{32}$

Consistently with our results, Lee et al observed that in subjects with high Hcy levels, the possibility of CAD risk was considerably increased. ${ }^{33}$ On the contrary, a large metaanalysis conducted to examine the connection of moderately increased levels of Hcy with CHD, which included 48,175 CHD patients and 67,961 controls, showed an insignificant impact on the risk of CHD. ${ }^{34}$ Cross-sectional studies have consistently shown that increased Hcy is correlated with $\mathrm{CVD}^{35}$ The plaques developed in coronary artery endothelial cells are more vulnerable to detachment by hyperhomocysteinemia. ${ }^{36}$ However, other studies have not confirmed such correlations. ${ }^{37,38}$

The role of Hcy in patients with atherosclerosis and associated vascular consequences has been extensively studied. The role of hyperhomocysteinemia and its correlation with CAD has been subjected to extensive research. ${ }^{2,39,40}$ Moderately increased Hcy $(>13-15 \mu \mathrm{mol} / \mathrm{L})$ has a multifactorial relationship with various genetic and environmental interactions and consequently cardiogenic effects. ${ }^{41}$

Our results confirm that elevated hs-CRP is correlated with an elevated risk of CAD. We also demonstrated that CAD is similarly related to Hcy and has a tendency toward an association with high TG and low HDL-C. Consistently with this, Seo et al found a significant association between hs-CRP and risk of CAD. ${ }^{11}$ This in agreement with previous studies illustrating that serum hs-CRP is an independent risk factor of CVD, regardless of LDL-C level. ${ }^{11,42,43}$ Moreover, hs-CRP may offer considerable additive value in predicting cardiovascular risks.

The inflammatory response is thought to play a major role in atherosclerosis formation and progression. Acute reactant hs-CRP appears to trigger endothelial dysfunction and promote inflammation in the arterial wall, resulting in atherogenesis and an elevated risk of cardiovascular events. ${ }^{11}$

Our results showed that patients with CAD had significantly higher levels of serum troponin I, in agreement with Jia et al, who found that cardiac troponin I was positively and strongly associated with incident CAD. ${ }^{14}$

Consistently, Lima et al found that in stable CAD patients, higher resting levels and elevation of highsensitivity troponin I with exercise were predictors of adverse cardiovascular outcomes beyond traditional cardiovascular risk factors and presence of inducible ischemia. $^{44}$ 

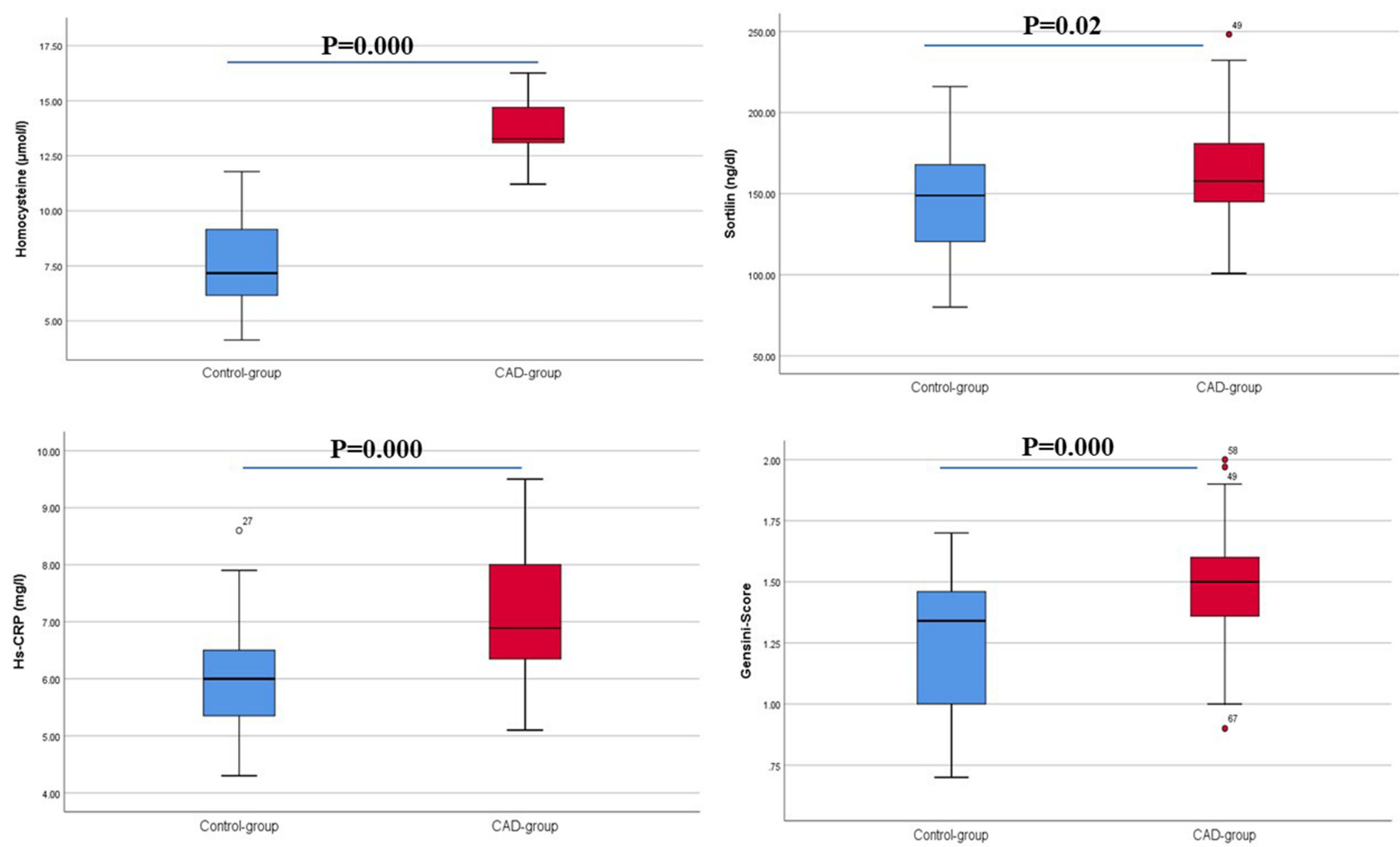

Figure 2 Differences in measured variables between the groups.
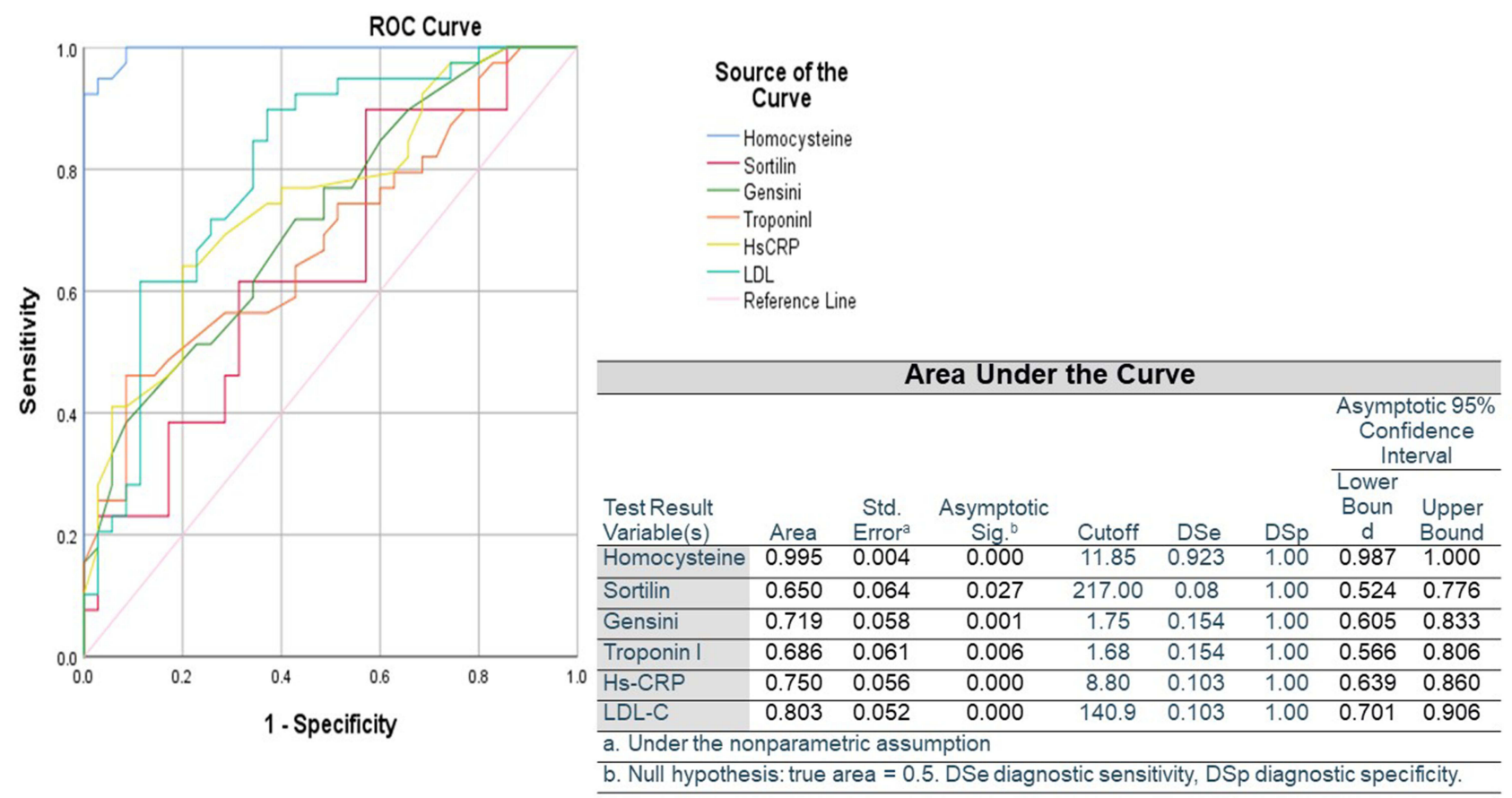

Figure 3 Area under ROC curve of the ability of measured variables to predict presence of coronary artery disease.

Similarly, Zhu et al found that cardiac troponin I was an independent predictor of fatal and non-fatal CVD events and could be used to identify individuals at risk in a general population. ${ }^{45}$
Our results showed a significant negative relationship between Hcy and HDL-C and a positive association between Hcy and angiographic Gensini scores. Also, ROC-curve analysis demonstrated that Hcy was the most sensitive biomarker 
Table 2 Correlations among Measured Parameters in Both Groups

\begin{tabular}{|c|c|c|c|c|c|c|c|c|c|}
\hline & & LDL & HDL & TG & Troponin I & Hs-CRP & Gensini & Homocysteine & Sortilin \\
\hline TC & $\begin{array}{l}r \\
P\end{array}$ & $\begin{array}{c}0.91 * * \\
0\end{array}$ & $\begin{array}{c}-0.34 * * \\
0.003\end{array}$ & $\begin{array}{c}0.63^{* *} \\
0\end{array}$ & $\begin{array}{c}0.61^{* *} \\
0\end{array}$ & $\begin{array}{c}0.58^{* * *} \\
0\end{array}$ & $\begin{array}{c}0.62 * * \\
0\end{array}$ & $\begin{array}{c}0.65^{* *} \\
0.02\end{array}$ & $\begin{array}{c}0.61^{* *} \\
0\end{array}$ \\
\hline LDL-C & $\begin{array}{l}r \\
P\end{array}$ & & $\begin{array}{c}-0.30 \text { ** } \\
0.009\end{array}$ & $\begin{array}{c}0.40 * * \\
0\end{array}$ & $\begin{array}{c}\left.0.4\right|^{* *} \\
0\end{array}$ & $\begin{array}{l}0.35^{* *} \\
0.002\end{array}$ & $\begin{array}{c}0.42^{* *} \\
0\end{array}$ & $\begin{array}{c}0.5 I^{* *} \\
0\end{array}$ & $\begin{array}{l}0.37^{* *} \\
0.001\end{array}$ \\
\hline HDL-C & $\begin{array}{l}r \\
P\end{array}$ & & & $\begin{array}{c}-0.31^{* *} \\
0.007\end{array}$ & $\begin{array}{c}-0.26^{*} \\
0.02\end{array}$ & $\begin{array}{c}-0.39 * * \\
0.00 \mathrm{I}\end{array}$ & $\begin{array}{c}-0.22 \\
0.06\end{array}$ & $\begin{array}{c}-0.42 * * \\
0\end{array}$ & $\begin{array}{c}-0.21 \\
0.08\end{array}$ \\
\hline TGs & $\begin{array}{l}r \\
P\end{array}$ & & & & $\begin{array}{c}0.85^{* *} \\
0\end{array}$ & $\begin{array}{c}0.83^{* *} \\
0\end{array}$ & $\begin{array}{c}0.91 * * \\
0\end{array}$ & $\begin{array}{c}0.72 * * \\
0\end{array}$ & $\begin{array}{c}0.91 * * \\
0\end{array}$ \\
\hline Troponin I & $\begin{array}{l}r \\
P\end{array}$ & & & & & $\begin{array}{c}0.74^{* *} \\
0\end{array}$ & $\begin{array}{c}0.85^{* *} \\
0\end{array}$ & $\begin{array}{c}0.58^{* * *} \\
0\end{array}$ & $\begin{array}{c}0.82^{* *} \\
0\end{array}$ \\
\hline $\mathrm{Hs}-\mathrm{CRP}$ & $\begin{array}{l}r \\
P\end{array}$ & & & & & & $\begin{array}{c}0.87^{* *} \\
0\end{array}$ & $\begin{array}{c}0.73^{* *} \\
0\end{array}$ & $\begin{array}{c}0.87^{* *} \\
0\end{array}$ \\
\hline Gensini & $\begin{array}{l}r \\
P\end{array}$ & & & & & & & $\begin{array}{c}0.7 I^{* *} \\
0\end{array}$ & $\begin{array}{c}0.93 * * \\
0\end{array}$ \\
\hline Homocysteine & $\begin{array}{l}r \\
P\end{array}$ & & & & & & & & $\begin{array}{c}0.32 * * \\
0.006\end{array}$ \\
\hline
\end{tabular}

Notes: $* * P<0.01$ (two-tailed); $* P<0.05$ (two-tailed).

Abbreviations: TC, total cholesterol; LDL-C, low density-lipoprotein cholesterol; HDL-C, high density-lipoprotein cholesterol; TGs, triglycerides; Hs-CRP, high-sensitivity CRP.

Table 3 Logistic Regression Analysis of Sortilin and Homocysteine as Independent Risk Factors of Coronary Artery Disease

\begin{tabular}{|l|c|c|c|c|}
\hline & \multirow{2}{*}{ OR } & \multicolumn{2}{|c|}{ 95\% CI } & \multirow{2}{*}{ P } \\
\cline { 3 - 4 } & & Lower & Upper & \\
\hline Homocysteine $(\mu \mathrm{mol} / \mathrm{L})$ & 27.503 & 1.521 & 497.233 & 0.025 \\
Sortilin $(\mathrm{ng} / \mathrm{dL})$ & 1.018 & 1.002 & 1.034 & 0.025 \\
\hline
\end{tabular}

Table 4 Logistic Regression Analysis of Effects of Statin and Aspirin Use and Associated Diseases in Coronary Artery Disease Patients

\begin{tabular}{|l|c|c|c|c|}
\hline \multirow{2}{*}{} & \multirow{2}{*}{ OR } & \multicolumn{2}{|c|}{ 95\% Cl } & \multirow{2}{*}{$P$} \\
\cline { 3 - 4 } & & Lower & Upper & \\
\hline Smoker & 0.644 & 0.197 & 2.106 & 0.467 \\
Statins & 0.592 & 0.218 & 1.605 & 0.303 \\
Aspirin & 0.512 & 0.178 & 1.467 & 0.213 \\
Hypertension & 1.138 & 0.335 & 3.874 & 0.836 \\
Diabetes & 0.663 & 0.237 & 1.860 & 0.435 \\
\hline
\end{tabular}

for CAD, followed by LDL-C, hs-CRP, Gensini score, and troponin-I, whereas sortilin was the least sensitive. We believe that these results emphasize the importance of recognizing individuals with elevated Hcy that could be at risk of atherothrombotic disease. So, focusing on preventive measures, such as intake of folate, vitamin $\mathrm{B}_{6}$, and $\mathrm{B}_{12}$, which are known to decrease Hcy levels, ${ }^{2}$ in order to define the role of Hcy and
Hcy-lowering therapy in reducing risk in high-risk CAD patients are thus required.

Our results showed that patients with CAD tended to have significantly higher sortilin concentrations than control subjects. In accordance with our results, Oh et $\mathrm{al}^{46}$ demonstrated that sortilin is a protein associated with CAD. A Japanese study, in contrast to our data, ${ }^{17}$ revealed that subjects with CAD had lower sortilin levels than nonCAD subjects. Other research has shown that sortilin plays a major role in cholesterol metabolism. ${ }^{16,47,48}$ Previous investigational data have proved that sortilin is implicated in atherosclerotic plaque formation by enhancing the inflammatory pathway in a mouse model. ${ }^{49}$

Reduced plasma HDL- and LDL-cholesterol associated with reduction of atherosclerotic lesions has been observed in whole-body sortilin-knockout mice ${ }^{16}$ and vascular calcification. ${ }^{50}$ Correspondingly, decreased atherosclerotic lesions due to decreased LDL uptake by macrophages have been displayed in macrophage sortilin-deficient mice. ${ }^{21}$ In contrast, serum LDL-cholesterol levels are diminished following exaggerated expression of sortilin in the liver. ${ }^{51}$ Our findings, in accordance with Ogawa et al, ${ }^{17}$ who demonstrated that sortilin levels were significantly associated with LDL-C, TG, hs-CRP, and Hcy levels, which are considered cardiovascular risk factors. HDL possesses potent antiinflammatory 
characteristics that may have a protective effect against atherogenesis. $^{52}$ Clinical and epidemiological studies have demonstrated that HDL concentration in atherosclerotic CVD is often negatively associated with plasma-CRP levels. ${ }^{53}$ The present study established that hs-CRP had a significant association with CAD severity evaluated using angiographic Gensini scores. This in consistent with Masood et al, who carried out a cross-sectional study to investigate the relationship between hs-CRP and coronary atherosclerosis severity on 80 patients subjected to coronary angiography. ${ }^{54}$

Moukarbel et al discovered that raised CRP were associated with coronary artery-lesion complexity. ${ }^{55}$ In terms of plaque ulceration and inflammation, attempts have also been made to relate the susceptibility of coronary plaques to serum hs-CRP levels. Espligureal et al observed considerably higher hs-CRP levels in patients suffering from acute coronary syndrome than patients with chronic stable angina. ${ }^{56}$ There has been confirmation that CRP is a strong predictor of CVD risk among patients undergoing elective revascularization treatment, patients with acute coronary syndromes, and even apparently healthy individuals. ${ }^{57}$ Peppes et $\mathrm{al}^{58}$ showed that serum levels of myocardial enzymes and inflammatory biomarkers not only increased at the time of an acute coronary event but also - according to angiographic results (Gensini score) - were quantitatively associated with the degree of myocardial damage and the severity of CAD. A negative correlation between HDL levels and the severity of CAD (Gensini score) was identified in this study and a reverse correlation between serum HDL and CRP was observed. The present study revealed a negative correlation between HDL level and Gensini score, the index of CAD severity in CAD patients and controls.

Wadham et al found that via oxidation of HDL, main phospholipid is neutralizing the proinflammatory ability of CRP in endothelial cells, showing an equilibrium between antiinflammatory and proinflammatory actions inside the vascular wall. Increased CRP concentration is often correlated with factors that reduce the amount and/or quality of HDL, such as age, diabetes, and obesity. ${ }^{59,60}$ This possibly will finally disrupt the anti-inflammatory and proinflammatory equilibrium to promote the progression of inflammatory CVD. Tarchalski et $\mathrm{al}^{61}$ who conducted a prospective study, showed that coronary atherosclerosis severity was positively associated with TC, LDL-C, and TGs and inversely associated with HDL-C in angina pectoris patients with no previous myocardial infarction.

Our data demonstrated that in Egyptian subjects, Hcy and sortilin levels may provide additive value in predicting cardiovascular risk, and interventions that reduce these levels may be effective in reducing the incidence of cardiovascular events.

\section{Limitations}

We had a relatively small sample size. In order to examine the potential clinical relevance of our results to management of CAD, a prospective study is needed.

\section{Conclusion}

Hcy and circulating sortilin are novel and major elevated biomarkers in patients with CAD, and may be used as tools for diagnosis and potential therapeutic targets in CAD patients. However, additional human studies are needed to confirm our results.

\section{Funding}

The authors did not get any funding or grant from any organizations.

\section{Disclosure}

All authors declare no conflict of interest.

\section{References}

1. Badimon L, Storey RF, Vilahur G. Update on lipids, inflammation and atherothrombosis. Thromb Haemost. 2011;105(S 06):S34-S42. doi:10.1160/THS10-11-0717

2. Baños-González MA, Anglés-Cano E, Cardoso-Saldaña G, et al. Lipoprotein (a) and homocysteine potentiate the risk of coronary artery disease in male subjects. Circ J. 2012:1204161700. doi:10.1253/circj. CJ-12-0039

3. von Scholten BJ, Reinhard H, Hansen TW, et al. Additive prognostic value of plasma $\mathrm{N}$-terminal pro-brain natriuretic peptide and coronary artery calcification for cardiovascular events and mortality in asymptomatic patients with type 2 diabetes. Cardiovas Diabetol. 2015;14 (1):59. doi:10.1186/s12933-015-0225-0

4. Kumar A, Palfrey HA, Pathak R, Kadowitz PJ, Gettys TW, Murthy SN. The metabolism and significance of homocysteine in nutrition and health. Nutr Metab. 2017;14(1):1-12. doi:10.1186/ s12986-017-0233-z

5. Gauthier GM, Keevil JG, McBride PE. The association of homocysteine and coronary artery disease. Clin Cardiol. 2003;26(12):563-568. doi:10.1002/clc.4960261204

6. Wald DS, Law M, Morris JK. Homocysteine and cardiovascular disease: evidence on causality from a meta-analysis. BMJ. 2002;325 (7374):1202. doi:10.1136/bmj.325.7374.1202

7. Schaffer A, Verdoia M, Cassetti E, et al. Relationship between homocysteine and coronary artery disease. Results from a large prospective cohort study. Thromb Res. 2014;134(2):288-293. doi:10.1016/j.thromres.2014.05.025

8. Vivona N, Bivona G, Noto D, et al. C-reactive protein but not soluble CD40 ligand and homocysteine is associated to common atherosclerotic risk factors in a cohort of coronary artery disease patients. Clin Biochem. 2009;42(1617):1713-1718. doi:10.1016/j.clinbiochem.2009.08.014

9. Boekholdt SM, Kastelein J. C-reactive protein and cardiovascular risk: more fuel to the fire. Lancet. 2009;375(9709):95-96. doi:10.1016/ S0140-6736(09)62098-5 
10. Kumakura H, Fujita K, Kanai H, et al. High-sensitivity C-reactive protein, lipoprotein (a) and homocysteine are risk factors for coronary artery disease in Japanese patients with peripheral arterial disease. $J$ Atheroscler Thromb. 2014:25478. doi:10.5551/jat.25478

11. Seo SM, Baek SH, Jeon HK, et al. Correlations between the level of high-sensitivity $\mathrm{C}$-reactive protein and cardiovascular risk factors in Korean adults with cardiovascular disease or diabetes mellitus: the CALLISTO study. $J$ Atheroscler Thromb. 2013;20(7):616-622. doi:10.5551/jat. 16089

12. Iribarren C, Chandra M, Rana JS, et al. High-sensitivity cardiac troponin I and incident coronary heart disease among asymptomatic older adults. Heart. 2016;102(15):1177-1182. doi:10.1136/heartjnl2015-309136

13. Omland T, Pfeffer MA, Solomon SD, et al. Prognostic value of cardiac troponin I measured with a highly sensitive assay in patients with stable coronary artery disease. J Am Coll Cardiol. 2013;61 (12):1240-1249. doi:10.1016/j.jacc.2012.12.026

14. Jia X, Sun W, Hoogeveen RC, et al. High-sensitivity troponin I and incident coronary events, stroke, heart failure hospitalization, and mortality in the ARIC study. Circulation. 2019;139(23):2642-2653. doi:10.1161/CIRCULATIONAHA.118.038772

15. Dube JB, Johansen CT, Hegele RA. Sortilin: an unusual suspect in cholesterol metabolism: from GWAS identification to in vivo biochemical analyses, sortilin has been identified as a novel mediator of human lipoprotein metabolism. Bioessays. 2011;33(6):430-437. doi:10.1002/bies.201100003

16. Kjolby M, Andersen OM, Breiderhoff T, et al. Sort1, encoded by the cardiovascular risk locus 1p13. 3, is a regulator of hepatic lipoprotein export. Cell Metab. 2010;12(3):213-223. doi:10.1016/j.cmet.2010. 08.006

17. Ogawa K, Ueno T, Iwasaki T, et al. Soluble sortilin is released by activated platelets and its circulating levels are associated with cardiovascular risk factors. Atherosclerosis. 2016;249:110-115. doi:10. 1016/j.atherosclerosis.2016.03.041

18. Strong A, Rader DJ. Sortilin as a regulator of lipoprotein metabolism. Curr Atheroscler Rep. 2012;14(3):211-218. doi:10.1007/s11883-0120248-x

19. Kjolby M, Nielsen MS, Petersen CM. Sortilin, encoded by the cardiovascular risk gene SORT1, and its suggested functions in cardiovascular disease. Curr Atheroscler Rep. 2015;17(4):18. doi:10.1007/ s11883-015-0496-7

20. Gao A, Cayabyab FS, Chen X, et al. Implications of sortilin in lipid metabolism and lipid disorder diseases. DNA Cell Biol. 2017;36 (12):1050-1061. doi:10.1089/dna.2017.3853

21. Patel KM, Strong A, Tohyama J, et al. Macrophage sortilin promotes LDL uptake, foam cell formation, and atherosclerosis. Circ Res. 2015;116(5):789-796. doi:10.1161/CIRCRESAHA.116.305811

22. Gensini GG. A more meaningful scoring system for determining the severity of coronary heart disease. Am J Cardiol. 1983;51(3):606 doi:10.1016/S0002-9149(83)80105-2

23. Elasfar A, Hekal S, ElGendy E, Abdulaal M, Elsokkary $\mathrm{H}$, Ashmawy M. Assessment of left and right atrial geometrical changes in patients with stable coronary artery disease: left and right atrial strain and strain rate imaging study. Egypt Heart J. 2018;70 (2):101-106. doi:10.1016/j.ehj.2018.02.003

24. Lilliefors HW. On the Kolmogorov-Smirnov test for normality with mean and variance unknown. $J$ Am Stat Assoc. 1967;62 (318):399-402. doi:10.1080/01621459.1967.10482916

25. Sanchis-Gomar F, Perez-Quilis C, Leischik R, Lucia A. Epidemiology of coronary heart disease and acute coronary syndrome. Ann Transl Med. 2016;4(13):13. doi:10.21037/atm.2016.06.33

26. De Luca G, Verdoia M, Suryapranata H. Benefits from intracoronary as compared to intravenous abciximab administration for STEMI patients undergoing primary angioplasty: a meta-analysis of 8 randomized trials. Atherosclerosis. 2012;222(2):426-433. doi:10.1016/j. atherosclerosis.2012.02.041
27. De Luca G, Ernst N, Van't Hof AW, et al. Predictors and clinical implications of early reinfarction after primary angioplasty for ST-segment elevation myocardial infarction. Am Heart J. 2006;151 (6):1256-1259. doi:10.1016/j.ahj.2005.06.047

28. De Luca G, Gibson CM, Bellandi F, et al. Diabetes mellitus is associated with distal embolization, impaired myocardial perfusion, and higher mortality in patients with ST-segment elevation myocardial infarction treated with primary angioplasty and glycoprotein IIb-IIIa inhibitors. Atherosclerosis. 2009;207(1):181-185. doi:10.10 16/j.atherosclerosis.2009.03.042

29. De Luca G, Van't Hof AW, Ottervanger JP, et al. Ageing, impaired myocardial perfusion, and mortality in patients with ST-segment elevation myocardial infarction treated by primary angioplasty. Eur Heart J. 2005;26(7):662-666. doi:10.1093/eurheartj/ehi110

30. McCully KS. Vascular pathology of homocysteinemia: implications for the pathogenesis of arteriosclerosis. Am J Pathol. 1969;56(1):111.

31. Clarke R, Daly L, Robinson K, et al. Hyperhomocysteinemia: an independent risk factor for vascular disease. New Engl J Med. 1991;324(17):1149-1155. doi:10.1056/NEJM199104253241701

32. Klerk M, Verhoef P, Clarke R, Blom HJ, Kok FJ, Schouten EG. MTHFR $677 \mathrm{C} \rightarrow \mathrm{T}$ polymorphism and risk of coronary heart disease: a meta-analysis. JAMA. 2002;288(16):2023-2031. doi:10.1001/jama. 288.16.2023

33. Lee B-J, Lin P-T, Liaw Y-P, Chang S-J, Cheng C-H, Huang Y-C. Homocysteine and risk of coronary artery disease: folate is the important determinant of plasma homocysteine concentration. Nutrition. 2003;19(7-8):577-583. doi:10.1016/S0899-9007(02)010 98-5

34. Clarke R, Bennett DA, Parish S, et al. Homocysteine and coronary heart disease: meta-analysis of MTHFR case-control studies, avoiding publication bias. PLoS Med. 2012;9(2):e1001177. doi:10.1371/ journal.pmed.1001177

35. Splaver A, Lamas GA, Hennekens CH. Homocysteine and cardiovascular disease: biological mechanisms, observational epidemiology, and the need for randomized trials. Am Heart J. 2004;148 (1):34-40. doi:10.1016/j.ahj.2004.02.004

36. Han K, Lu Q, Zhu W, Wang T, Du Y, Bai L. Correlations of degree of coronary artery stenosis with blood lipid, CRP, Hcy, GGT, SCD36 and fibrinogen levels in elderly patients with coronary heart disease. Eur Rev Med Pharmacol Sci. 2019;23(21):9582-9589.

37. Bozkurt A, Toyaksi H, Acartürk E, Tuli A, Çayli M. The effects of hyperhomocysteinemia on the presence, extent, and severity of coronary artery disease. Jpn Heart J. 2003;44(3):357-368. doi:10.1536/ jhj.44.357

38. Brilakis ES, McConnell JP, Ballman KV, Klee GG, Berger PB. Lack of association between plasma homocysteine and angiographic coronary artery disease in the era of fortification of cereal grain flour with folic acid. Atherosclerosis. 2002;165(2):375-381. doi:10.1016/ S0021-9150(02)00303-9

39. Akyürek Ö, Akbal E, Güneş F. Increase in the risk of ST elevation myocardial infarction is associated with homocysteine level. Arch Med Res. 2014;45(6):501-506. doi:10.1016/j.arcmed.2014.08.003

40. Cioni G, Marcucci R, Gori AM, et al. Increased homocysteine and lipoprotein (a) levels highlight systemic atherosclerotic burden in patients with a history of acute coronary syndromes. J Vasc Surg. 2016;64(1):163-170. doi:10.1016/j.jvs.2016.01.056

41. Masud R, Baqai HZ. The communal relation of MTHFR, MTR, ACE gene polymorphisms and hyperhomocysteinemia as conceivable risk of coronary artery disease. App Physiol Nutr Metab. 2017;42 (10):1009-1014. doi:10.1139/apnm-2017-0030

42. Koenig W, Sund M, Fröhlich M, et al. C-reactive protein, a sensitive marker of inflammation, predicts future risk of coronary heart disease in initially healthy middle-aged men: results from the MONICA (Monitoring Trends and Determinants in Cardiovascular Disease) Augsburg cohort study, 1984 to 1992. Circulation. 1999;99 (2):237-242 
43. Xu M, Bi Y, Chen Y, et al. Increased C-reactive protein associates with elevated carotid intima-media thickness in Chinese adults with normal low density lipoprotein cholesterol levels. J Atheroscler Thromb. 2013;20(6):575-584. doi:10.5551/jat.15172

44. Lima BB, Hammadah M, Kim JH, et al. Relation of high-sensitivity cardiac troponin i elevation with exercise to major adverse cardiovascular events in patients with coronary artery disease. Am J Cardiol. 2020;136:1-8. doi:10.1016/j.amjcard.2020.09.019

45. Zhu K, Knuiman M, Divitini M, et al. High-sensitivity cardiac troponin I and risk of cardiovascular disease in an Australian population-based cohort. Heart. 2018;104(11):895-903. doi:10.1136/heartjnl-2017-312093

46. Oh TJ, Ahn CH, Kim B-R, et al. Circulating sortilin level as a potential biomarker for coronary atherosclerosis and diabetes mellitus. Cardiovas Diabetol. 2017;16(1):92. doi:10.1186/s12933-017-0568-9

47. Gustafsen C, Kjolby M, Nyegaard M, et al. The hypercholesterolemia-risk gene SORT1 facilitates PCSK9 secretion. Cell Metab. 2014;19(2):310-318. doi:10.1016/j.cmet.2013.12.006

48. Linsel-Nitschke P, Samani NJ, Schunkert H. Sorting out cholesterol and coronary artery disease. New Engl J Med. 2010;363(25):2462. doi:10.1056/NEJMcibr1010765

49. Mortensen MB, Kjolby M, Gunnersen S, et al. Targeting sortilin in immune cells reduces proinflammatory cytokines and atherosclerosis. J Clin Invest. 2014;124(12):5317-5322. doi:10.1172/JCI76002

50. Goettsch C, Hutcheson JD, Aikawa M, et al. Sortilin mediates vascular calcification via its recruitment into extracellular vesicles. $J$ Clin Invest. 2016;126(4):1323-1336. doi:10.1172/JCI80851

51. Musunuru K, Strong A, Frank-Kamenetsky M, et al. From noncoding variant to phenotype via SORT1 at the $1 \mathrm{p} 13$ cholesterol locus. Nature. 2010;466(7307):714-719. doi:10.1038/nature09266

52. Cockerill GW, Rye K-A, Gamble JR, Vadas MA, Barter PJ. Highdensity lipoproteins inhibit cytokine-induced expression of endothelial cell adhesion molecules. Arterioscler Thromb Vasc Biol. 1995;15 (11):1987-1994. doi:10.1161/01.ATV.15.11.1987
53. Ridker PM, Hennekens CH, Buring JE, Rifai N. C-reactive protein and other markers of inflammation in the prediction of cardiovascular disease in women. New Engl J Med. 2000;342(12):836-843. doi:10.1056/NEJM200003233421202

54. Masood A, Jafar SS, Akram Z. Serum high sensitivity C-reactive protein levels and the severity of coronary atherosclerosis assessed by angiographic gensini score. Atherosclerosis. 2011;3:4.

55. Moukarbel GV, Arnaout MSA, Alam SE. C-reactive protein is a marker for a complex culprit lesion anatomy in unstable angina. Clin Cardiol. 2001;24(7):506-510. doi:10.1002/clc.4960240718

56. Arroyo-Espliguero R, Avanzas P, Cosín-Sales J, Aldama G, Pizzi C, Kaski JC. C-reactive protein elevation and disease activity in patients with coronary artery disease. Eur Heart J. 2004;25(5):401-408. doi:10.1016/j.ehj.2003.12.017

57. Brunetti ND, Troccoli R, Correale M, Luigi Pellegrino P, Di BM. $\mathrm{C}$-reactive protein in patients with acute coronary syndrome: correlation with diagnosis, myocardial damage, ejection fraction and angiographic findings. Int J Cardiol. 2006;109(2):248-256. doi:10.1016/j. ijcard.2005.06.021

58. Peppes V, Rammos G, Manios E, Koroboki E, Rokas S, Zakopoulos N. Correlation between myocardial enzyme serum levels and markers of inflammation with severity of coronary artery disease and Gensini score: a hospital-based, prospective study in Greek patients. Clin Interv Aging. 2008;3(4):699. doi:10.2147/CIA.S3551

59. Chambers JC, Eda S, Bassett P, et al. C-reactive protein, insulin resistance, central obesity, and coronary heart disease risk in Indian Asians from the United Kingdom compared with European whites. Circulation. 2001;104(2):145-150. doi:10.1161/01.CIR.104.2.145

60. Wadham C, Albanese N, Roberts J, et al. High-density lipoproteins neutralize C-reactive protein proinflammatory activity. Circulation. 2004;109(17):2116-2122. doi:10.1161/01.CIR.0000127419.45975.26

61. Tarchalski J, Guzik P, Wysocki H. Correlation between the extent of coronary atherosclerosis and lipid profile. Mol Cell Biochem. 2003;246(1-2):25-30. doi:10.1023/A:1023443609730
International Journal of General Medicine

\section{Publish your work in this journal}

The International Journal of General Medicine is an international, peer-reviewed open-access journal that focuses on general and internal medicine, pathogenesis, epidemiology, diagnosis, monitoring and treatment protocols. The journal is characterized by the rapid reporting of reviews, original research and clinical studies across all disease areas. The manuscript management system is completely online and includes a very quick and fair peer-review system, which is all easy to use. Visit http://www.dovepress.com/ testimonials.php to read real quotes from published authors. 